\title{
Rol de la TC multicorte con RMP en el diagnóstico de la hernia obturatriz. Presentación de un caso
}

Dres. Juan Carlos Ignacio Larsson ${ }^{(1)}$, Gregorio Hernández(1), Marco Marangoni C(2), Prof. Alberto Marangoni C(2).

1. Servicio de Cirugía General del Sanatorio Allende, Córdoba - Argentina.

2. Servicio de Diagnóstico por Imágenes del Sanatorio Allende, Córdoba - Argentina.

Role of Multislice CT with MPR (Multi-planar reformation) in the diagnosis of obturator hernia. A case report.

\begin{abstract}
The obturator hernia $(\mathrm{OH})$ is rare and accounts for 0.05 to $0.4 \%$ of all hernias. Because of its rarity and its nonspecific presentation, diagnosis is usually late and has high mortality rates (12-70\%). A typical case of $\mathrm{OH}$ diagnosed preoperatively by Multislice CT with MPR and treated using emergency laparotomy with good results, is presented. The use of Multislice CT with MPR of the abdomen and pelvis, in symptoms of intestinal obstruction in elderly women with no history of previous surgery or objectified hernias, has great value for early preoperative diagnosis of $\mathrm{OH}$ and could help reduce the high rates of morbidity and mortality.

Keywords: Early diagnosis, Multislice CT, Obturator hernia.
\end{abstract}

Resumen: La hernia obturatriz $(\mathrm{HO})$ es poco frecuente y representa del 0.05 al $0.4 \%$ del total de hernias. Debido a su rareza y a su presentación inespecífica, el diagnóstico es por lo general tardío y las tasas de mortalidad elevadas (12-70\%). Se presenta un caso típico de HO diagnosticada en el preoperatorio mediante TC Multicorte con RMP y tratado mediante laparotomía de urgencia con buen resultado. El empleo de TC Multicorte con RMP de abdomen y pelvis, en cuadros de obstrucción intestinal en mujeres añosas, sin antecedente de cirugías previas ni hernias objetivables, tiene gran valor para el diagnóstico preoperatorio precoz de HO y podría contribuir a reducir las elevadas tasas de morbilidad y mortalidad.

Palabras clave: Diagnóstico temprano, Hernia obturatriz, TC multicorte.

Larsson JC, et al. Rol de la TC Multicorte con RMP en el diagnóstico de la hernia obturatriz. Presentación de un caso. Rev Radiol 2014; 20(1): 21-25.

Correspondencia: juanlarsson@hotmail.com

Trabajo recibido el 13 de marzo de 2013. Aceptado para publicación el 03 de marzo de 2014.

\section{Introducción}

La hernia obturatriz $(\mathrm{HO})$ es poco frecuente y representa del 0.05 al $0.4 \%$ del total de hernias y del 0.2 al $1.6 \%$ de los casos de obstrucción de intestino delgado ${ }^{(1-4)}$. Debido a su rareza y a su presentación inespecífica, el diagnóstico es por lo general tardío(2) y las tasas de mortalidad elevadas $(12-70 \%)^{(1,4-5)}$. La Tomografía Computada Multislice (TCM) con reconstrucción multiplanar (RMP), contribuye al diagnóstico preoperatorio precoz ${ }^{(6)}$. Se presenta un caso típico de $\mathrm{HO}$ diagnosticada en el preoperatorio mediante TC y tratado mediante laparotomía de urgencia con buen resultado.

\section{Reporte del caso}

Una mujer de 80 años multípara con antecedentes personales patológicos de hipertensión arterial, car- diopatía isquémica, enfermedad pulmonar obstructiva crónica y cifoescoliosis fue internada por cuadro de abdomen agudo suboclusivo de 2 días de evolución y deshidratación. No presentaba antecedentes quirúrgicos abdominales.

El examen físico reveló emaciación importante y un abdomen distendido, timpánico y doloroso, sin peritonismo ni hernias palpables en la región inguinal. Tampoco presentó signo de Howship-Romberg.

El examen de laboratorio al ingreso evidenció

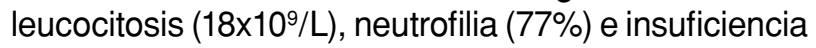
renal aguda (urea: $203 \mathrm{mg} / \mathrm{dl}$, creatinina: $3.5 \mathrm{mg} / \mathrm{dl}$, potasio: 5,2 mequiv/L).

En la radiografía directa de abdomen de pie se observaron asas intestinales distendidas con niveles hidroaéreos y escasa cantidad de aire en el marco colónico (Figura 1). 

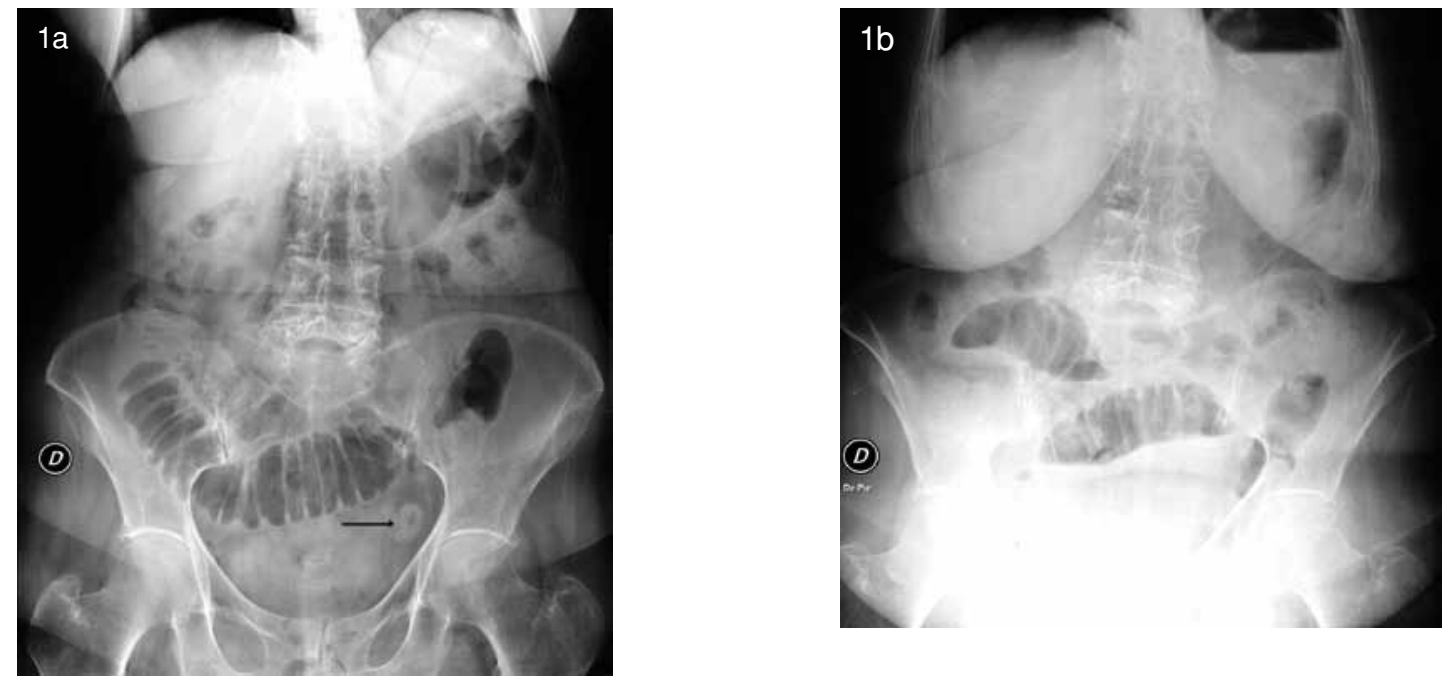

Figura 1a,b. Directa de abdomen obtenida en decúbito (a) y en posición de pie (b). Distensión de asas de intestino delgado en ambas proyecciones, con niveles hidroaéreos en la posición vertical, con escaso aire en el marco colónico. Puede objetivarse como signo indirecto discutible, la presencia de un engrosamiento de un asa tomada de frente, tangencialmente, de probable edema parietal luego objetivado en la TCM (Flecha en a).

El tratamiento médico inicial durante las primeras 12 horas de internación, mediante descompresión nasogástrica e hidratación endovenosa, permitió el descenso de los valores de creatinina sérica $(2,6 \mathrm{mg} / \mathrm{dl})$, buen ritmo de diuresis (50 $\mathrm{ml} /$ hora), pero sin mejoría franca del cuadro abdominal. Ante este cuadro y conociendo los valores de creatinina del paciente de 2 meses previos normales $(0,8 \mathrm{mg} / \mathrm{dl})$ se realizó TCM con RMP abdomen pelviana, con contraste endovenoso, con el consentimiento del paciente. La misma demostró una hernia obturatriz izquierda incarcerada (Figura 2). En la Figura 3, se identificaron signos de sufrimiento del asa intestinal afectada, lo que motivo la indicación de cirugía de urgencia.

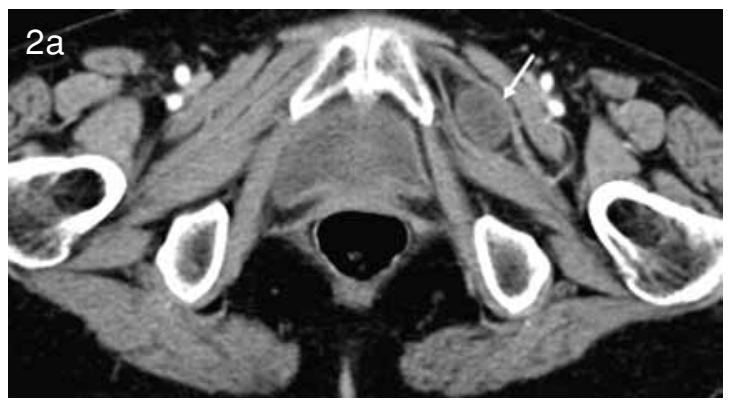

Figura 2a,b,c. TC Multicorte sin ingestión de contraste y con inyección de sustancia yodada e.v., en planos axial (a), coronal (b) y sagital (c). (a) Imagen redondeada, con pequeño nivel hidroaéreo entre los músculos obturador y pectíneo (flecha blanca). (b) Distensión de asas de intestino delgado, sin dilatación del colon. Se observa un asa en posición anómala, proyectada por debajo del isquion izquierdo (flechas blancas); en b, asa intestinal pre herniaria con pared congestiva edematosa y reforzada con el contraste e.v., coincidente con la imagen de la $R x$ (flecha corta). (c) Asa intestinal en el saco herniario (flecha blanca).
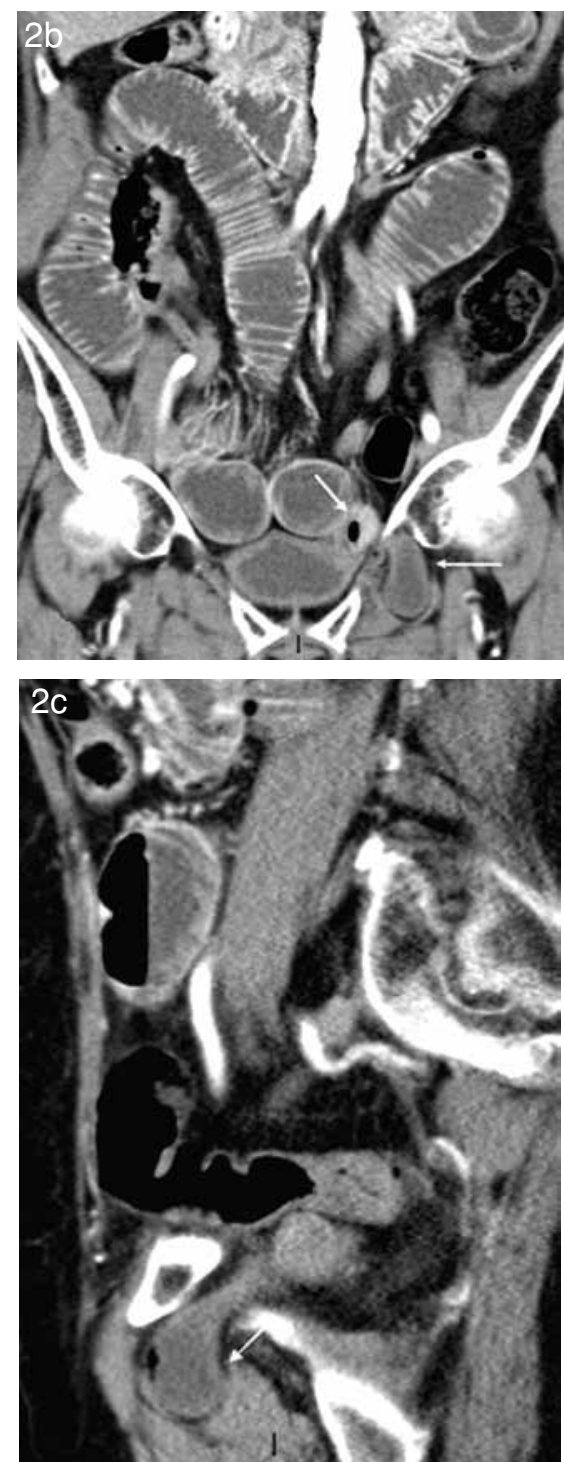


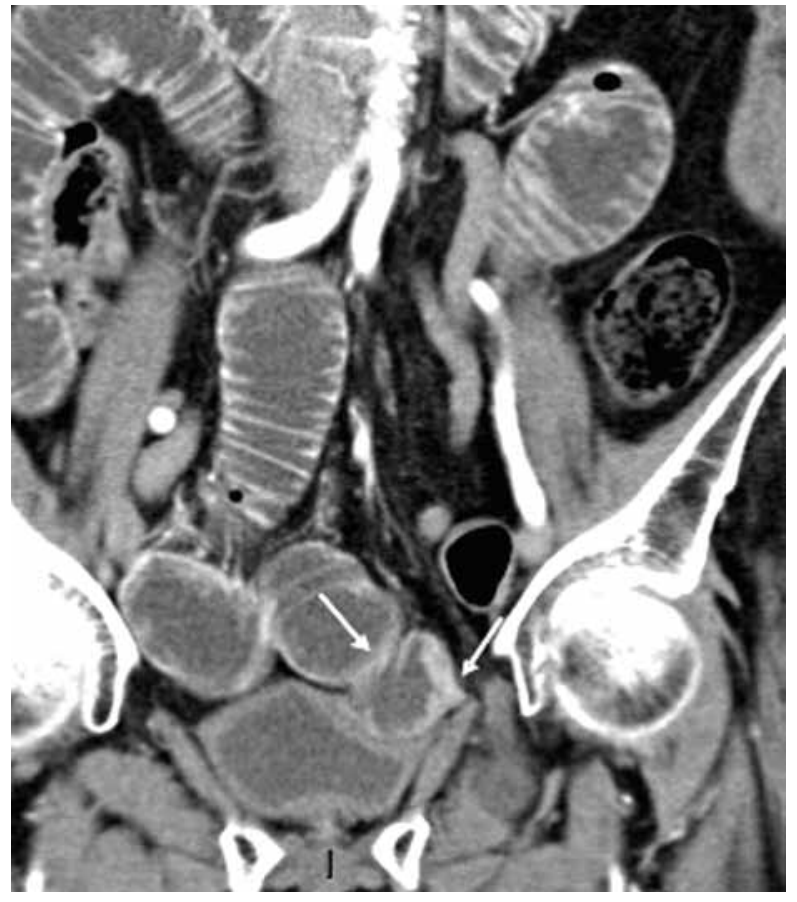

Figura 3. TC Multicorte sin ingestión de contraste y con inyección de sustancia yodada e.v., en plano coronal. Identificación de asas pre herniarias con edema parietal, signo de sufrimiento, con realce pos contraste e.v. (fechas).

Durante la laparotomía de urgencia, se evidenció una hernia tipo Richter en el foramen obturador izquierdo, que se redujo manualmente (Figura 4). Se resecó el segmento intestinal no viable y se resolvió la hernia ligando el saco a nivel del orificio obturador con puntos simples. El pos operatorio cursó con recuperación completa de la función renal, sin necesidad de hemodiálisis ni complicaciones asociadas otorgándose el alta hospitalaria al décimo día. La paciente continúa asintomática después de un seguimiento de 8 meses.

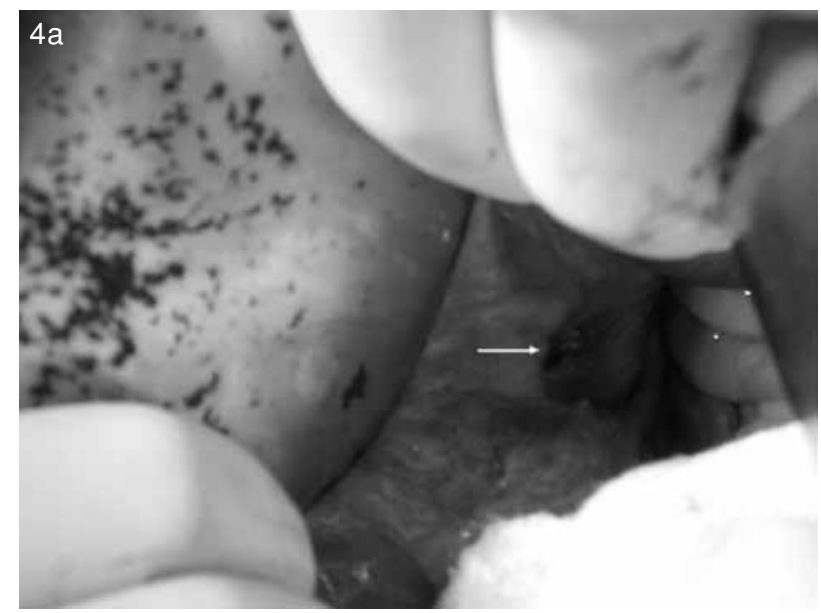

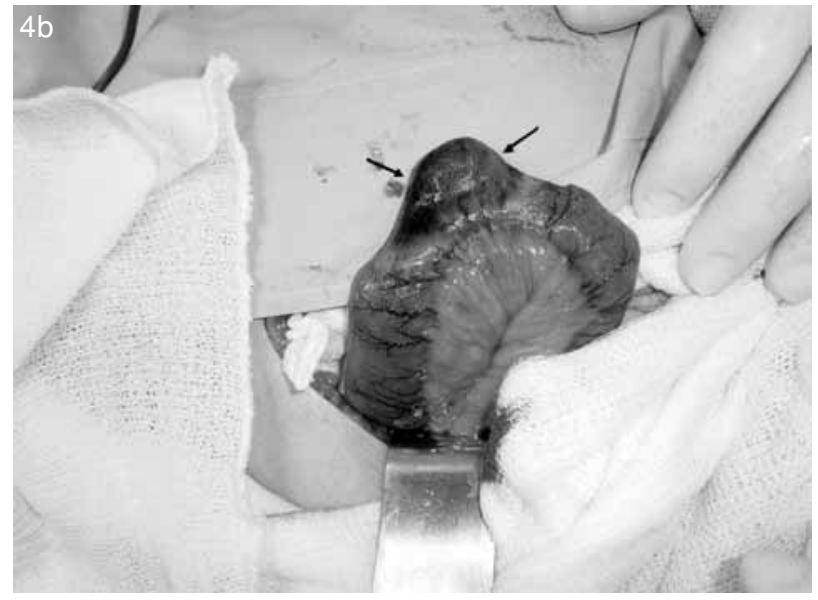

Figura 4a,b. Cirugía. (a) Visualización del orificio herniario (flecha). (b) Asa intestinal con signos de sufrimiento vascular (fechas).

\section{Discusión}

La hernia obturatriz fue descrita por primera vez por Le Marie en 1718, aunque otros postulan que el primero en hacerlo fue Ronsil en el año $1724^{(4)}$, y desde entonces han sido reportados cerca de 800 casos $^{(3,4)}$.

Se la conoce como "hernia de la anciana delgada", ya que predomina en mujeres emaciadas multíparas con una media de edad de 80 años $^{(6)}$. El lado derecho es el más afectado, debido a la situación del colon sigmoides del lado izquierdo, que actúa como diafragma $^{(4,5)}$. El porcentaje de bilateralidad va del 6 al $63 \%{ }^{(7,8)}$ y un $13 \%-88 \%$ se asocia a hernias femorales $^{(2,8)}$. Normalmente, la hernia contiene intestino delgado ${ }^{(3)}$ y el porcentaje de hernias de Richter se aproxima al $70 \%{ }^{(5)}$.

Los tres tipos de hernia obturatriz incluyen ${ }^{(3)}$ (Figura 5):

Tipo 1: el saco atraviesa al canal obturador y se localiza entre el músculo obturador externo y el pectíneo, siendo el hallazgo más frecuente;

Tipo 2: el saco se exterioriza a través de las fibras superiores e inferiores del músculo transverso cubierta por el pectíneo superficialmente; $y$

Tipo 3: el saco queda alojado entre ambos músculos obturadores, (rara).

El diagnóstico es un verdadero desafío incluso para médicos experimentados, debido a su clínica inespecífica y a su bajo índice de sospecha ${ }^{(1)}$. El diagnóstico preoperatorio ocurre sólo en el 10$31.3 \%$ de los $\operatorname{casos}^{(1,4,5)}$, ya que por lo general, se realiza durante una laparotomía exploradora por obstrucción intestinal o peritonitis ${ }^{(1)}$. El retraso en la indicación quirúrgica aumenta la necesidad de resección intestinal $(25-75 \%)^{(6)}$, en pacientes con escasa reserva fisiológica por su edad avanzada, malnutrición y comorbilidades asociadas ${ }^{(5)}$, lo que eleva considerablemente la mortalidad $(12-70 \%)^{(1,4-6)}$. 


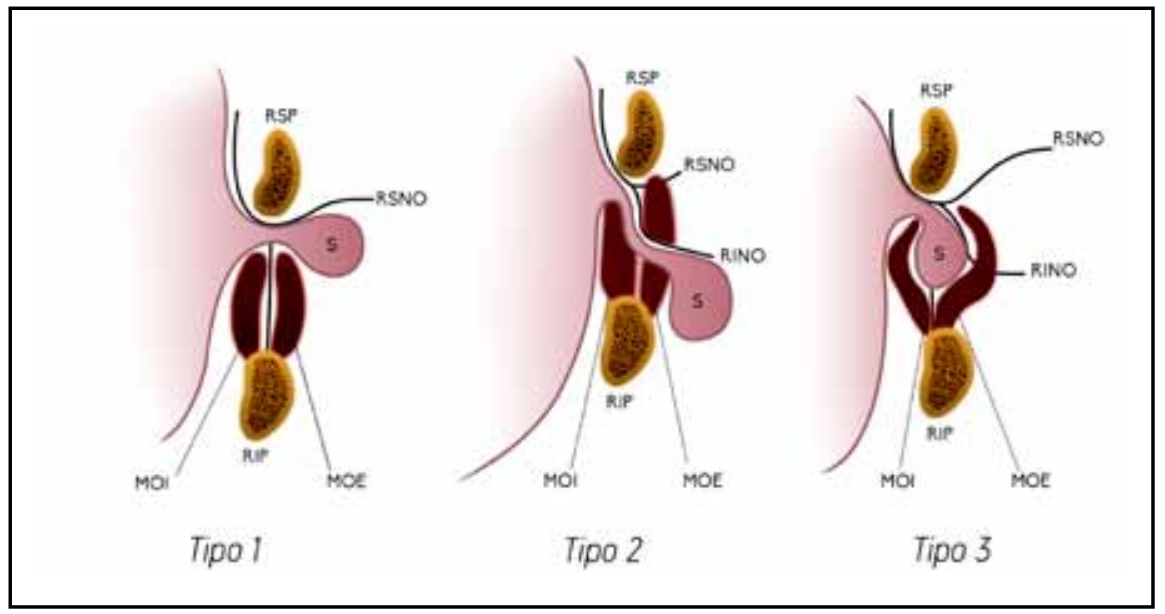

Figura 5. Clasificación de la hernia obturatriz en tres tipos. RSNO: Rama Superior del Nervio Obturador; RINO: Rama Inferior del Nervio Obturador; MOI: Músculo Obturador Externo; MOI: Músculo Obturador Externo; RSP: Rama Superior del Pubis; RIP: Rama Inferior del Pubis; y SH: Saco Herniario

Existen tres signos clínicos específicos de hernia incarcerada. El primero es la neuralgia del nervio obturador, ocasionada por la compresión del paquete vásculo nervioso y manifestada por calambres o hipo-hiperestesia constante o intermitente en la ingle irradiados a la cara anterointerna del muslo( ${ }^{(9)}$. El segundo es el Signo de Howship-Romberg (HR) que aparece entre el 15 y el $50 \%$ de los pacientes, y se caracteriza por síntomas de neuralgia provocados por la extensión, abducción y rotación interna de la cadera ${ }^{(5,9)}$. Este puede quedar enmascarado por el dolor abdominal concomitante y no es patognomónico, ya que suele confundirse con dolor osteoarticular a nivel de la cadera en la población geriátrica ${ }^{(1,4)}$. El tercero es el Signo de Hannington-Kiffque consiste en ausencia del reflejo aductor, en presencia de reflejo patelar ipsilateral ${ }^{(3)}$. Este signo es menos conocido, pero más específico ${ }^{(3)}$. Rara vez puede palparse la hernia a nivel del agujero obturador, ya que la $\mathrm{HO}$ se encuentra en la profundidad del músculo pectíneo ${ }^{(3)}$. El tacto vaginal puede ser de utilidad en estos casos, palpando en hora $2 \mathrm{u}$ hora $10^{(9)}$.

Por último, la oclusión intestinal es el síntoma cardinal de presentación en el $90 \%$ de los $\operatorname{casos}^{(4,9)}$, ya sea de forma aguda o intermitente ${ }^{(1)}$. Este signo debería ser evaluado rápidamente con estudios por imágenes para prevenir la demora en el diagnóstico y es aquí donde la TC juega su rol más importante. Se requiere de un alto índice de sospecha de parte del radiólogo al momento de informar el estudio, ya que la hernia estrangulada puede ser evidenciada por primera vez en la TC.

La utilidad de la TC de abdomen y pelvis fue reportada por primera vez por Cubillo en el año $1983^{(5)}$ y su utilización cada vez más frecuente permite que una proporción mayor de pacientes sean diagnosticados en el preoperatorio(6). Actualmente constituye la modalidad diagnóstica gold standard(6), con una sensibilidad del $78 \%$ al $100 \%{ }^{(3,6)}$ y una eficacia diagnóstica del $80-100 \%^{(2,9)}$. La TC con RMP agrega información importante a la otorgada por las imágenes axiales, ya que mejora la visualización del defecto herniario delineando con más detalle el saco y sus complicaciones ${ }^{(10)}$. Los tres tipos de hernia obturatriz pueden ser evidenciados mediante $\mathrm{TC}^{(3)}$, aunque generalmente ésta demuestra una masa hipodensa localizada entre el obturador externo y el pectíneo (tipo 1) $)^{(7)}$.

En este reporte de caso presentamos una mujer añosa, delgada y multípara, sin antecedentes quirúrgicos, en quién la única pista de hernia obturatriz estrangulada era un cuadro suboclusivo intestinal confirmado mediante radiografía abdominal. La TCM permitió un diagnóstico etiológico temprano mostrando en forma clara y precisa el sitio de la hernia y los signos de sufrimiento intestinal, lo cual permitió un tratamiento oportuno y una recuperación rápida.

Existe más evidencia que apoya nuestra creencia de que el diagnóstico temprano mediante TC, seguido de laparotomía de urgencia podría reducir las tasas de morbilidad y mortalidad ${ }^{(2,3,5,8)}$, en pacientes que tal vez hubiesen sido tratados inicialmente de manera conservadora. Sin embargo, es importante considerar lo dicho por otros autores: "la hernia obturatriz necesita de una cirugía y no de un diagnóstico", para subrayar la necesidad de exploración quirúrgica inmediata en presencia de peritonitis u oclusión intestinal, sin solicitar estudios de imágenes innecesarios que sólo retrasarían el tratamiento oportuno ${ }^{(1,4,5)}$. La TC está indicada en aquellos pacientes que se presentan con cuadro oclusivo intestinal sin urgencia quirúrgica y sin etiología evidente por el examen clínico, ya que permite el diagnóstico de $\mathrm{HO}$, y la evaluación de signos de 
incarceración o estrangulación que determinarán la necesidad de exploración quirúrgica de urgencia ${ }^{(1)}$.

En conclusión, a pesar de la baja incidencia de hernia obturatriz en la población general, ésta debe ser considerada como causa probable de obstrucción intestinal en mujeres añosas emaciadas, sin antecedente de cirugías previas ni hernias objetivables. La mortalidad todavía permanece alta como consecuencia del retraso en el diagnóstico y la escasa reserva fisiológica de estos pacientes. El diagnóstico preoperatorio mediante TCM con RMP abdominopelviana, podría contribuir a reducirla. Para ello, es necesario que los médicos tomografistas tengan presente la posibilidad de $\mathrm{HO}$ en todos los casos de oclusión intestinal, cuando la causa no sea visualizada en la cavidad abdominal.

\section{Bibliografía}

1. Chang SS, Shan YS, Lin YJ, Tai YS, Lin PW. A review of obturator hernia and a proposed algorithm for its diagnosis and treatment. World J Surg 2005; 29: 450454.

2. Mantoo SK, Mak K, Tan TJ. Obturator hernia: Diagnosis and treatment in the modern era. Singapore Med J 2009; 50: 866-870.
3. Stamatiou D, Skandalakis LJ, Zoras O, Mirilas P, Surg M. Obturator Hernia Revisited: Surgical Anatomy, Embryology, Diagnosis and Technique of Repair. Am Surg 2011; 77: 1147-1157.

4. Rodríguez-Hermosa JI, Codina-Cazador A, MarotoGenover A, Puig-Alcántara J, Sirvent Calvera JM, Garsot-Savall E, et al. Obturator hernia: clinical analysis of 16 cases and algorithm for its diagnosis and treatment. Hernia 2008: 12: 289-297.

5. Igari K, Ochiai T, Aihar A, Kumagai Y, Lida M, Yamazaki S. Clinical presentation of obturator hernia and review of the literatura. Hernia 2010; 14: 409-413.

6. Sun HP, Chao YP. Preoperative diagnosis and successful laparoscopic treatment of incarcerated obturator hernia. Hernia 2010; 14: 203-206.

7. Basnet RB, Rijal B, Sharma VK. Abstruse Diagnosis of Obturator Hernia - A Case Series. J Nepal Med Assoc 2010; 50: 316-319.

8. Yokoyama T, Kobayashi A, Kikuchi T, Hayashi K, Miyagawa S. Transabdominal Preperitoneal Repair for Obturator Hernia. World J Surg 2011; 35: 2323-2327.

9. Losanoff JE, Richman BW, Jones JW. Obturator hernia. J Am Coll Surg 2002; 194: 657-663.

10. Aguirre DA, Santosa AC, Casola G, Sirlin CB. Abdominal Wall Hernias: Imaging Features, Complications, and Diagnostic Pitfalls at Multi-Detector Row CT. Radiographics 2005; 25: 1501-1520. 